\title{
Enhancing Innovation through Virtual Proximity Tom Coughlan
}

\author{
"No distance of a place or lapse of time can lessen the") \\ friendship of those who are thoroughly persuaded of \\ each other's worth.
}

Robert Southey (1774-1843)

Poet, scholar, and historian

\begin{abstract}
Historically, innovation strategists have focused on leveraging local resources and the development of local clusters, which have relied heavily on personal contact. It was assumed that serendipity would occur through casual contact and that this contact would result in rapid sharing of ideas. Many studies have supported this concept; however, the pace of innovation has changed and the most successful organizations promote not only physical proximity but also virtual proximity to resources. Virtual proximity refers to the level of emotional closeness between individuals, as developed through the use of information and communications technologies. This article argues that organizations can and should look to develop local virtual relationships supported by physical proximity: the mix of both virtual proximity and physical proximity can increase an organization's innovation capability.
\end{abstract}

\section{Introduction}

Ever since Samuel Morse tapped out "What hath God wrought?" on his telegraph to send the first electronic message (Howe, 2007; tinyurl.com/m8n724a), and with the release of every information communications technology since, there have been pundits who have proclaimed the "death of distance"(e.g., Bowersox and Calantone, 1998: tinyurl.com/m5rkxx2; Cairncross, 1997: tinyurl.com/m7sqhsc; Evans and Harrigan, 2005: tinyurl.com/ ld2xjnu). But, to paraphrase another nineteenth century luminary, Mark Twain (tinyurl.com/57mptu), the reports of its death have been greatly exaggerated. Distance is still alive and well and creating havoc for those of us who practice or study innovation.

Even though we can tap out a text, send an email, make a phone call, or share in a video conference, part of the message is lost if we are not sharing the same physical location with the people on our innovation team. Just moving the location of a key person or resource a few metres can dramatically drop the level of interaction and therefore amount of innovation an organization will produce (Allen, 2007; tinyurl.com/lshbss7). But, the sharing of information is not just about physical distance - it is about a shared connection. To truly under- stand these connections, and in turn how innovation happens, it is important to understand the concepts of proximity, effective communication, information architecture, and some of the properties of the media used for intra-organizational communications.

Managers, entrepreneurs, researchers, and innovators of all types need to find new ways of leveraging both their existing resources and discovering new potential innovation resources. Innovation is often a function of recombining ideas and resources that often already exist or building on the ideas of others - who may exist both inside and outside your organization (Kelley, 2005; tinyurl.com/1440oal). Many studies have supported the notion that casual, serendipitous contact facilitates idea sharing (Bindroo et al., 2012: tinyurl.com/mmh5t58; Hauser et al., 2007: tinyurl.com/qdk4dhf; Huggins and Izushi, 2011: tinyurl.com/plnnt9a; Knoben and Oerlemans, 2006: tinyurl.com/kn3svq9; Porter, 1990: tinyurl.com/khf32f4); but, unfortunately, given the pace of modern lifestyles, our ability to travel, and the required commitments of many of our potential collaborators, it is often difficult if not impossible to be in the same place at the same time. However, some level of proximity is necessary in order for ideas to collide and serendipity to occur. Therefore, we need to develop a new virtual type of 


\section{Enhancing Innovation through Virtual Proximity}

Tom Coughlan

proximity that allows our collaborators to be aware of the new ideas or potential resources - and this awareness could lead to the development of a feeling of presence and possibly engagement, which increases the likelihood of innovation.

This article focuses on virtual proximity as a means of enhancing innovation. To understand the problems that make virtual proximity an important part of an innovation strategy, it is critical to understand some of the key principles surrounding it; therefore, this article will be structured as follows. First, the different types of proximity and their roles are identified. Next, the key elements of effective communications and media use are examined, and the key factors surrounding regional clusters and their effect on innovation are outlined. Then, some of the misconceptions surrounding virtual proximity are dispelled. Finally, a foundation for a solid virtual proximity strategy is provided, along with some simple and actionable recommendations for managers.

\section{Proximity}

Proximity to resources, and the clustering of resources by specific industries within a geographic region, has long been considered an important factor in the promotion of both the volume and the quality of innovation (Doloreux, 2004: tinyurl.com/k7botqn; Porter, 2001: tinyurl.com $/ \mathrm{kp21808).} \mathrm{The} \mathrm{belief} \mathrm{is} \mathrm{that} \mathrm{close} \mathrm{geographic}$ proximity to key resources would reduce friction and speed access to those resources and therefore increase innovation. Some researchers have gone as far as to suggest that tacit knowledge is an essential ingredient of innovation, and that tacit knowledge can only be transferred in close physical proximity. The true value of clustering emerges when proximity of both key resources and tacit knowledge fosters the spillover of knowledge within and across industries (Greunz, 2003: tinyurl.com/pefatjs; Knoben and Oerlemans, 2006: tinyurl.com/kn3svq9).

This perspective, however, begs the questions: what is proximity? The definition of proximity dramatically changed when Wilfred Beckerman (1956; tinyurl.com/ lyjhhyx) introduced the term psychic distance. Beckerman's contention was that distance is not an absolute. The distance between two individuals is a function of the disparity of their cultures, not the physical distance between them. The concept of psychic distance has been expanded by a number of researchers, leading to the development of additional concepts such as:
- cultural proximity: how similar the cultures of network participants are on a national level (Hofstede, 2009: tinyurl.com/5p6sme; Knoben and Oerlemans, 2006: tinyurl.com/kn3svq9; Sousa and Bradley, 2006: tinyurl.com/n2na6by)

- cognitive distance: the level of diversity in the skills, knowledge, and cognitive frame (Wuyts et al., 2005; tinyurl.com/khvb7ca)

- organizational proximity: the distance felt by members of the same large or multi-site organization (Knoben and Oerlemans, 2006: tinyurl.com/kn3svq9)

- technology proximity: the level of overlap between the firms' technology or patent portfolio

- vision proximity: the similarity in vision (Cantu, 2010; tinyurl.com/prlxkb4)

- virtual proximity: the level of emotional closeness developed through the use of information and communications technologies (Coughlan, 2010; tinyurl.com/ olqrel7)

These descriptions of proximity are not mutually exclusive, it is often unclear where they begin and end, and there are gradient scales to each and every one. For example, even geographic proximity, one of the most straightforward of the proximity metrics, can be measured in either physical distance or travel time. Some researchers have gone as far as to develop meta indexes that attempt to combine several of these elements into a single measure of proximity (Amin and Cohendet, 2005: tinyurl.com/k6ebtry; Coughlan, 2010: tinyurl.com/olqrel7). So, defining how close you are to a resource can be more difficult than what might be originally assumed.

\section{Communications}

The principles of proximity, culture, and cognition have a dramatic effect on the encoding, transmission, decoding, and processing of an idea from one individual to another. However, when understanding the strategy of communication, it is just as important to understand the "what and why" (i.e., the architecture) of the communications. Allen (2007; tinyurl.com/lshbss7) suggested that relationships within the organization affect the success of the communications, and that there are three types of communications, each of which is affected by its own proximity or relationship dynamics: 


\section{Enhancing Innovation through Virtual Proximity}

Tom Coughlan

1. Type I: simple communications required to coordinate group or team projects.

2. Type II: the sharing of codified knowledge.

3. Type III: the transfer of tacit knowledge, which is the most important type of knowledge for innovation and the one most affected by distance.

Allen's study also found that, unsurprisingly, people who work in close physical proximity to each other will typically communicate more often than those who do not. However, what was surprising is that, when this relationship is plotted on a curve, little to no drop in the level of communications can be seen beyond 50 metres. Allen posited that visual clues to a person's existence are important in prompting communications.

\section{Media}

In order for innovation teams to properly communicate key ideas, their choice of communication media is often extremely important. Each medium has inherent properties and limitations; as we increase the distance between team members - and reduce the time they are physically co-present - the importance of this choice increases. Media richness theory (Lengel and Daft, 1988; tinyurl.com/ogd2k2v) posits that performance of communications improves with the richness of the communications media. For example, phone conversations are richer than text messages, and videoconferences are richer than phone calls. In addition, as the equivocality of the task increases, so should the richness of the media used (Lengel and Daft, 1988; tinyurl.com/ogd2k2v). After decades of study what has been discovered is that real communications often transcends the media (Dennis and Kinney, 1998; tinyurl.com/kw6qf8y): our successful use of media is often dependent on our familiarity with that media and our familiarity with the recipient of the message. Another key finding is that the less natural we feel in using a media, the more cognitive resources we will need to expend (Dennis et al., 2008; tinyurl.com/ mk9w6c7). However, with time and effort, our familiarity with a specific medium improves and the cognitive effort declines (Dennis et al., 2008; tinyurl.com/mk9w6c7).

\section{Anatomy of Clusters}

According to Porter (1998; tinyurl.com/38rnvv6), clusters are "geographic concentrations of interconnected companies and institutions in a particular field." Porter's work has often been cited as seminal in terms of outlining the concepts of clusters and why cluster provide a competitive advantage in efficiency and innovation. Porter points out that clusters often provides a company with access to employees, suppliers, specialized information, and key services that are difficult and more expensive to obtain outside the cluster. The clusters that have historically worked best have clear industry foci and many inter-organizational relationships, allowing that the advantages become specialized to a specific industry or the needs of a particular type of customer (Porter, 1998; tinyurl.com/38rnvv6). But, can we supplement the advantages that geographic proximity delivers through access to non-local resources? Would some other form of proximity, such as virtual proximity or cultural proximity, provide an even greater competitive advantage? For example, in comparing California's Silicon Valley to the Route 128 Corridor in Massachusetts, there is a cultural difference in how innovation has historically been handled. Although both regions are focused on the technology Industry, Silicon Valley has been much more open to inter-organizational relationships and sharing; resulting in a far more dramatic regional growth (Saxenian, 1994; tinyurl.com/m3xzkjq). Knoben (2008; tinyurl.com/14400al) demonstrated that it is not just about the density of firms or the size of the population; the success of innovation is dependent on the membership of the internal team as well as the connections and relationships developed outside the firm. The makeup of the regional economy has a strong influence on local success: "...simply bringing firms together, for example by building science parks, is unlikely to effectively stimulate the innovativeness of firms and might even hamper it" (Knoben, 2008; tinyurl.com/1440oal). The cluster of firms must have a culture and a resource profile that not only allows but also encourages each firm to interact (Ben Letaifa and Rabeau, 2013; tinyurl.com/ pjx9yj3). Virtual proximity might help fill a gap in a team's talent profile with a person or firm that has a better cultural fit than a local resource.

Studies by the author in the New York metropolitan area, have shown that firms that have a portfolio of inter-organizational relationships, which include both local and non-local linkages, are typically more innovative (Coughlan, 2010; tinyurl.com/olqrel7). In addition, top performers have inter-organizational relationship portfolios that are very broad in terms of the types of firms and industries included (Coughlan, 2010: tinyurl.com/ olqrel7; Knoben, 2008: tinyurl.com/1440oal). However, it is possible for a portfolio to be too broad. It is important that cognitive distance "be restricted for the sake of coordination" (Wuyts et al., 2005; tinyurl.com/khvb7ca). Diversity in thought is critical in innovation, but in this case you can have too much of a good thing. If a plot 


\section{Enhancing Innovation through Virtual Proximity}

Tom Coughlan

were developed to show innovation initiative over a scale of novelty and understandability an inverse Ushaped curve would develop. Too little diversity limits the available intellectual capital and too much diversity makes it difficult for team members to cognitively process the available information. So, organizations should be looking for a balance.

\section{Virtual Proximity}

Given this environment, virtual proximity can be a useful model if properly applied. However, there are a number of misconceptions or misunderstandings of how or when it should be leveraged - or even what it is.

Virtual proximity is about leveraging information and communications technologies to build and maintain relationships - the emphasis being on the relationship and not the technology. Simply having or using technology does not necessarily equate to an improvement in virtual proximity. Here, it might be important to think about the factors that nullified media richness theory, such as the familiarity with specific media tools and how cognitive ease improve with use (Dennis and Kinney, 1998; tinyurl.com/kw6qf8y). It is in the use of the technology and the integration into our work processes that we experience the advantages of virtual proximity. Once the use of a tool becomes familiar and easy to use, we can free up cognitive resources to work on innovation.

However, if the use of virtual proximity tools feels unnatural, too much of the cognitive effort will be devoted to the use of the tool and not into the content needed to develop the relationship or reorganizing of ideas and resources to develop new innovations. Although we can learn to use the tools and platforms, thereby reducing allocation of cognitive effort to the technology, we may struggle to keep up with the growth and change in these technologies and platforms. We want the latest technology, but we also want familiarity and efficacy.

It is tempting to assume that virtual proximity is primarily used to engage resources or individuals that exist outside the local region, and that it is not required for local relationships. However, this assumption is false. Allen (2007; tinyurl.com/lshbss7) points out that the probability of using a resource drops for every metre of separation up to 50 metres. Thus, the notion of "non local" starts at 50 metres. He also suggests that often we need visual clues to remind us that the resource is there. In- creasing the number of visual clues or contacts should help in reminding the network of the existence of a resource, and increase the probability of it being integrated into the innovation process.

Virtual proximity is multidimensional. Measuring virtual proximity requires the development of a matrix, which includes a variety of different electronic media, the level of use, the proficiency, and the impact of the use. In some way, it is similar to the concept of the Klout score (klout.com), which measures the influence of a given user across social media. However, there is no claim that a virtual proximity measure is an absolute measure. It is intended to be a model for thinking, just as one would use the product lifecycle in marketing or Tuckman's stages of group development in management (tinyurl.com/2bpowb4). As with these models, there are generalities that do apply. For example, a high degree of virtual proximity does generally result in higher level of innovation and higher levels of disruptive or intersectional innovation (Coughlan, 2010; tinyurl.com/ olqrel7).

Virtual proximity is similar to the notion of mental processing of social presence on the Internet, which has been described by Ning Shen and Khalifa (2008; tinyurl.com/lg14by2) as:

"...the moment-by-moment awareness of the copresence of another sentient being accompanied by a sense of engagement with the other... as a global, momentby-moment sense of the other, social presence is an outcome of cognitive stimulations (i.e., inferences) of the other's cognitive, emotional, and behavioral dispositions".

Whereas social presence emphasizes the real-time awareness of a resource's presence, virtual proximity emphasizes the ongoing awareness of a resource's existence. The key difference is that virtual proximity does not require engagement until the point it is integrated into the innovation process. In a sense, virtual proximity is more an awareness of the resource and the ability to readily engage the resource.

Virtual proximity is also different from the other forms of proximity outlined earlier in this article. However, it can act as a catalyst to improve other types of proximity such as psychic distance, cultural proximity, cognitive distance, and organizational proximity - all of which are broader concepts and span both the virtual and terrestrial worlds. 


\section{Enhancing Innovation through Virtual Proximity}

\section{Tom Coughlan}

\section{Conclusions}

Virtual proximity is not a choice - just as your reputation is not a choice. It exists in relative terms to the environment in which you live and operate. Individually or as an organization, we have a level of virtual proximity with every team member, supplier, partner, or collaborator that we currently have or could potentially have. However, just as your reputation can be managed and improved with time, vigilance, and effort, so can your virtual proximity. Managers should realize that the majority of telecommunications traffic is local - whether it be phone calls, text messages, tweets, emails, Facebook posts, Linkedin requests, Vines, or what whatever means of virtual communications your organization or network participates in. Virtual proximity is a local phenomenon.

In addition, the engagement levels of resources drop significantly in a matter of a few metres and the old adage "out of sight, out of mind" is constantly eroding our ability to stay aware of the resources and maintain our relationships. Virtual technologies are powerful tools that allow us to maintain our relations whether they are within our own organizations, across the street, or on the other side of the world.

Therefore, managers looking to capitalize on their innovation opportunities should have a proximity strategy. At a minimum, this strategy should include the following:

1. Visual clues: if at all possible, visual clues should be incorporated for key resources. Examples include making sure that photographs in social media profiles are up to date and that regular posts remind key resources of your existence. Simple tools that show presence are also important. Instant messaging tools such as Google Hangouts or Microsoft Lync could remind potential collaborators of each other's existence.

2. Combined proximities: as stated earlier, the effect that diversity has on innovation can be plotted as an inverse U-shaped curve. So, we need to find resources that have some minimal level of proximity on multiple scales of proximity (i.e., cultural, cognitive, organizational, technological, or vision), and we should engage resource outside the firm to help bolster the diversity of thought.
3. Common tools: it is important to develop familiarity with tools that enable virtual proximity. Virtual proximity can be developed using tools as simple as SMS or as complex as telepresence conference rooms; however, it is important that the users feel comfortable with whatever tools are chosen. Some of these tools will require training and all will require practice to use them properly without excessive cognitive effort. So, there must be some agreement, whether overt or implied, as to which tools will be used and why.

4. Regular integration of new tools: new tools are constantly being introduced in this area; however, managers must be careful in how they are integrated. New tools may have a technical advantage but the advantage might be negated but the additional overhead that it takes to be competent with a new tool. The introduction of too many new tools, or tools that feel unnatural to the users, could actually be a detriment to the process. Conversely, not introducing new capabilities that would improve the communications process and improve the level of virtual proximity could have the same effect.

5. Roll out of new tools with closely knit teams: given that familiarity with both the tools and the participants is important in reducing the cognitive overhead, when possible, new tools should be first introduced to participants who are familiar with each other. This approach will reduce the cognitive overhead and allow faster integration of the tool into the innovation process with the least disruption.

6. Experimentation: virtual proximity is a broad principle with few hard edges. It is likely that many of the key variables that surround virtual proximity will change over time and so will the specifics of virtual proximity. However, it is likely that the innovators will need to find power tools to maintain a broad set of relationships and expand their reach working with new collaborators and resources. In this sense, virtual proximity will likely increase in importance over time, and it will be necessary to develop new skills and techniques and capabilities in this area as our existing tools and techniques complete their lifecycle. 


\section{Enhancing Innovation through Virtual Proximity}

Tom Coughlan

\section{About the Author}

Tom Coughlan, DBA, is the Associate Dean of the School of Business at Mercy College in Dobbs Ferry, New York. He is also is an adjunct faculty in the graduate programs of the University of Phoenix, the Manhattan Institute of Management, the University of Bridgeport, and the Weller International Business School in Paris. His fields of practice include management, marketing, and e-business with a particular emphasis on the development of virtual proximity to increase levels of applied innovation within and across organizations. In addition to his academic activities, Dr. Coughlan has over 30 years of field experience as an entrepreneur, consultant, and marketing/management professional.

Citation: Coughlan, T. 2014. Enhancing Innovation

through Virtual Proximity. Technology Innovation

(cc) BY

Management Review. February 2014: 17-22.

Keywords: virtual proximity, innovation,

communication, distance, clusters, tools 